\title{
Turbulence fingerprint on collective oscillations of supernova neutrinos
}

\author{
Sajad Abbar๔ \\ Max-Planck-Institut für Physik (Werner-Heisenberg-Institut), Föhringer Ring 6, \\ 80805 München, Germany
}

(Received 28 July 2020; accepted 5 February 2021; published 23 February 2021)

\begin{abstract}
We bring to light a novel mechanism through which turbulent matter density fluctuations can induce collective neutrino flavor conversions in core-collapse supernovae, i.e., the leakage of flavor instabilities between different Fourier modes. The leakage mechanism leaves its notable fingerprint on the flavor stability of a dense neutrino gas by coupling flavor conversion modes on different scales which in turn, makes the flavor instabilities almost ubiquitous in the Fourier space. This intriguing phenomenon arises from the fact that unlike the case of collective neutrino oscillations in a homogenous medium, the neutrino flavor conversion modes depend linearly on each other at different supernova zones in a turbulent medium. The most remarkable consequence of this effect is in that it allows for the presence of significant flavor conversions in the deepest supernova regions even in the absence of the so-called fast modes. This is yet another crucial impact of turbulence on the physics of core-collapse supernovae which can profoundly change our understanding of neutrino flavor conversions in the supernova environment.
\end{abstract}

DOI: $10.1103 /$ PhysRevD.103.045014

\section{INTRODUCTION}

Core-collapse supernova (CCSN) explosions are among the most energetic astrophysical phenomena in which neutrino emission is a major effect $[1,2]$. Neutrino flavor evolution in CCSNe is a very rich and nonlinear phenomenon in which neutrinos can experience collective oscillations due to the high density of the ambient neutrino gas in the SN environment [3-6]. In this paper, we study collective neutrino oscillations in the presence of SN turbulent matter density fluctuations which as discussed later herein, can significantly impact the physics of neutrino oscillations in CCSNe.

Collective neutrino oscillations could significantly impact the physics of CCSNe. On the one hand, it could influence the SN dynamics and the nucleosynthesis of heavy elements [7] in the SN environment by modifying the neutrino and antineutrino energy spectra and consequently, their interaction rates. On the other hand, understanding of collective neutrino oscillations is crucial for future observations of galactic CCSNe neutrino signals $[8,9]$ and the upcoming measurements of diffuse supernova neutrino background [10].

The first studies on collective neutrino oscillations in $\mathrm{CCSNe}$ were carried out in maximally symmetric models,

Published by the American Physical Society under the terms of the Creative Commons Attribution 4.0 International license. Further distribution of this work must maintain attribution to the author(s) and the published article's title, journal citation, and DOI. Open access publication funded by the Max Planck Society. e.g., the stationary spherically symmetric neutrino bulb model [4-6,11-15]. Within these simplistic models it was observed that the onset of collective neutrino oscillations can be at radii much smaller than that of the conversions induced by ordinary matter via the Mikheyev-SmirnovWolfenstein (MSW) mechanism (at least in CCSNe with iron cores). Despite this, collective oscillations was still found to be suppressed in very deep $\mathrm{SN}$ regions due to the presence of high neutrino/matter densities [16-18]. However, it was then realized that in multidimensional (multi-D) time-dependent SN models, these suppressions can be dismissed thanks to the breaking of spatial/temporal symmetries [19-29]. Yet, in any realistic SN model, the physical conditions change so quickly that any unstable mode becomes stable before neutrinos can experience significant flavor conversions [25]. This means that in spite of the existence of flavor instabilities, significant flavor conversions should be unlikely to occur in the deepest regions of the $\mathrm{SN}$ core.

Nevertheless, it was then perceived that neutrinos can also experience the so-called fast flavor conversions on scales much shorter than those of traditional (slow) modes [30-51]. The fast scales are determined by the neutrino number density, $n_{\nu}$, and can be as short as a few $\mathrm{cm}$ in the deepest SN zones, as opposed to the ones of slow modes which are determined by the vacuum frequency, $\omega=\Delta m^{2} / 2 E$, and occur on scales of $\sim$ a few $\mathrm{km}$ (for $\Delta m_{\mathrm{atm}}^{2}$ and $E=10 \mathrm{MeV}$ neutrinos). Besides their phenomenological importance, perhaps the most remarkable physical consequence of fast modes is in that they can lead to the occurrence of collective neutrino oscillations in the 
deepest regions of the SN core. This is because they occur on short enough scales in such a way that the unstable modes can experience significant flavor conversions before the physical conditions vary significantly. In spite of their importance, fast modes do not seem to be a generic feature of CCSNe and even if they exist, they are thought to be present only in a finite region of the SN core [52-57]. Additionally, fast modes may also be less likely to occur in nonexploding SN models [58].

Turbulence plays a crucial role in CCSNe [59-63]. The impact of turbulent density fluctuations on neutrino oscillations has been extensively studied in 1D models [64-74], where it can induce flavor conversions through parametric resonances. Here, we demonstrate that the presence of turbulence in CCSNe can also induce collective neutrino flavor conversion modes via an entirely different mechanism, i.e., the leakage of flavor instabilities between different Fourier modes. This novel effect can significantly influence neutrino flavor evolution in the SN environment and in particular, it can lead to the presence of traditional (slow) collective neutrino oscillations in the deepest SN regions even in the absence of fast modes. What makes this novel effect more promising is in that it survives even for tiny turbulence amplitudes.

\section{LINEAR STABILITY ANALYSIS}

We start by deriving the equation of neutrino flavor evolution in the linear regime, in the two-flavor scenario where the flavor content of $a$ neutrino can be described as

$$
\varrho=\frac{f_{\nu_{e}}+f_{\nu_{x}}}{2}+\frac{f_{\nu_{e}}-f_{\nu_{x}}}{2}\left[\begin{array}{cc}
s & S \\
S^{*} & -s
\end{array}\right],
$$

where $f_{\nu}$ 's are the neutrino initial occupation numbers and, $S$ and $s$ carry information on neutrino flavor coherence and conversion, respectively. In the absence of collisions, the flavor evolution of the neutrino gas can be described by the Liouville-von Neumann equation $(c=\hbar=1)$ [75-79]

$$
i\left(\partial_{t}+\mathbf{v} \cdot \boldsymbol{\nabla}\right) \varrho_{E, \mathbf{v}}=\left[\frac{\mathrm{M}^{2}}{2 E}+\frac{\lambda}{2} \sigma_{3}+\mathrm{H}_{\nu \nu, \mathbf{v}}, \varrho_{E, \mathbf{v}}\right],
$$

where $\mathbf{v}$ is the neutrino velocity and $\lambda=\sqrt{2} G_{\mathrm{F}} n_{e}$ is the matter contribution to the neutrino Hamiltonian [80,81]. Here, the energies and occupation numbers are taken to be positive for neutrinos and negative for antineutrinos, $\sigma_{3}$ is the third Pauli matrix and

$$
\mathrm{H}_{\nu \nu, \mathbf{v}}=\sqrt{2} G_{\mathrm{F}} \int_{-\infty}^{\infty} \frac{E^{\prime 2} \mathrm{~d} E^{\prime}}{(2 \pi)^{3}} \int \mathrm{d} \mathbf{v}^{\prime} \varrho_{E^{\prime}, \mathbf{v}^{\prime}}\left(1-\mathbf{v} \cdot \mathbf{v}^{\prime}\right)
$$

is the contribution from the neutrino-neutrino forward scattering [82-84].
We are here interested in the flavor stability analysis of neutrinos in the linear regime where the flavor conversion is still insignificant and one has $s \simeq 1$ and $|S| \ll 1$. By only keeping terms of $\mathcal{O}(|S|)$ in Eq. (2), one reaches [85,86]

$$
i\left(\partial_{t}+\mathbf{v} \cdot \boldsymbol{\nabla}\right) S_{E, \mathbf{v}}=\left(\omega+\lambda+\Lambda_{\nu \nu, \mathbf{v}}\right) S_{E, \mathbf{v}}-h_{\nu \nu, \mathbf{v}},
$$

where, with the definition $g_{E, \mathbf{v}}=2 \varrho_{E, \mathbf{v}}^{00}(t=0)$,

$$
\begin{aligned}
& h_{\nu \nu, \mathbf{v}}=\sqrt{2} G_{\mathrm{F}} \int_{-\infty}^{\infty} \frac{E^{\prime 2} \mathrm{~d} E^{\prime}}{(2 \pi)^{3}} \int \mathrm{d} \mathbf{v}^{\prime} g_{E^{\prime}, \mathbf{v}^{\prime}} S_{E^{\prime}, \mathbf{v}^{\prime}}\left(1-\mathbf{v} \cdot \mathbf{v}^{\prime}\right), \\
& \Lambda_{\nu \nu, \mathbf{v}}=\sqrt{2} G_{\mathrm{F}} \int_{-\infty}^{\infty} \frac{E^{\prime 2} \mathrm{~d} E^{\prime}}{(2 \pi)^{3}} \int \mathrm{d} \mathbf{v}^{\prime} g_{E^{\prime}, \mathbf{v}^{\prime}}\left(1-\mathbf{v} \cdot \mathbf{v}^{\prime}\right) .
\end{aligned}
$$

Equation (4) provides a linear set of equations for which one can try collective solutions of the form $S_{E, \mathbf{v}}=$ $Q_{E, \mathbf{v}}^{\Omega, \mathbf{k}} e^{-i \Omega t+i \mathbf{k} \cdot \mathbf{x}}$ where $\Omega$ and $\mathbf{k}$ satisfy the dispersion relation (DR) equation corresponding to Eq. (4). In a homogenous medium, this leads to

$$
\left(-\Omega+\mathbf{v} \cdot \mathbf{k}+\omega+\lambda+\Lambda_{\nu \nu, \mathbf{v}}\right) Q_{E, \mathbf{v}}^{\Omega, \mathbf{k}}=h_{\nu \nu, \mathbf{v}}^{\Omega, \mathbf{k}} .
$$

Note that different Fourier modes are decoupled which means that $\mathbf{k}$ is just a parameter here and one only needs to find the functional form of $Q_{E, \mathbf{v}}^{\Omega, \mathbf{k}}$ in the $E-\mathbf{v}$ space for a solution of the DR equation.

\section{TURBULENT MATTER FLUCTUATIONS}

It simply follows from Eq. (6) that in a homogenous medium where matter is constant, the matter potential $\lambda$ can be absorbed in the real part of $\Omega$ and therefore, does not affect the stability condition of the dense neutrino gas. However, if the matter is not constant and spatial density fluctuations are present, Eq. (6) changes to

$$
\begin{aligned}
& \left(-\Omega+\mathbf{v} \cdot \mathbf{k}+\omega+\Lambda_{\nu \nu, \mathbf{v}}\right) Q_{E, \mathbf{v}}^{\Omega, \mathbf{k}} \\
& \quad+\int \frac{\mathrm{d}^{3} \mathbf{k}^{\prime}}{(2 \pi)^{3}} \lambda_{k^{\prime}} Q_{E, \mathbf{v}}^{\Omega, \mathbf{k}-\mathbf{k}^{\prime}}=h_{\nu \nu, \mathbf{v}}^{\Omega, \mathbf{k}},
\end{aligned}
$$

where $\lambda_{\mathbf{k}}$ is the Fourier component of the matter potential [87]. Note that, most remarkably, different Fourier modes are now coupled through the turbulence-induced convolution term and simple plane waves are not anymore eigenvectors of Eq. (7). This implies that in order to solve Eq. (7), one should also consider the distribution of $Q_{E, \mathbf{v}}^{\Omega, \mathbf{k}}$ in the Fourier space because $\mathbf{k}$ is not a parameter anymore and eigenvectors of Eq. (7) can now have contributions from a range of $\mathbf{k}$ 's.

In the following, we assume a Kolmogorov-like spectrum for turbulence where the matter density features power-law fluctuations [88] on a range of scales between the dissipation scale, $\lambda_{\text {diss }}$ (here $\lambda_{\text {diss }} \ll 10^{-10} \mathrm{~km}$ [59]), 
below which the turbulent energy gets efficiently dissipated by viscosity and the cutoff scale, $\lambda_{\text {cut }}$, which is determined by the shock radius $R_{\mathrm{S}}$ so that $\lambda_{\text {cut }} \sim 2 R_{\mathrm{s}}$.

To be specific, we take the turbulent matter fluctuations to have the form

$$
\lambda(x)=\lambda_{0}\left(1+\mathcal{C} \sum_{k \neq 0} \xi_{k} \cos \left(k x+\eta_{k}\right)\right),
$$

where $\lambda_{0}$ is the average matter potential (the zeroth mode), $\eta_{k}$ is a random phase and $\mathcal{C}=\mathfrak{C} / \mathfrak{C}_{\mathrm{N}}$ with $\mathfrak{\mathfrak { C }}$ and $\mathfrak{E}_{\mathrm{N}}$ being a constant coefficient and a normalization factor defined as $\mathfrak{C}_{\mathrm{N}}=\left(\sum_{k \neq 0} \xi_{k}^{2}\right)^{1 / 2}$, respectively. Here $\mathcal{C}$ is the most meaningful parameter which specifies the relative turbulence amplitude on scales $\sim \lambda_{\text {cut }}$. In addition, $\xi_{k}$ is assumed to have a Kolmogorov distribution

$$
\xi_{k}=\left(\frac{k}{k_{\mathrm{cut}}}\right)^{-\alpha / 2},
$$

with $k_{\text {cut }}=2 \pi / \lambda_{\text {cut }}$ which is fixed to be $k_{\text {cut }}=0.01 \mathrm{~km}^{-1}$ in our calculations. We also set $\alpha=5 / 3$ though our results do not depend qualitatively on the value of $\alpha$ for reasonable choices. With these choices, one has $\lambda_{k} \sim \mathcal{C} \lambda_{0}\left(k / k_{\text {cut }}\right)^{-\alpha / 2}$.

\section{TWO-BEAM MODEL}

We study neutrino flavor instabilities in a stationary 2D two-beam, monochromatic neutrino gas studied first in Ref. [21] (this stationary model is chosen for illustrative purposes, otherwise see Appendix B for the turbulence effect on temporal instabilities). Such a model can be used to describe the SN geometry at some distance from the SN core [25] where a periodic boundary condition is imposed in the transverse plane (along the $x$-axis in our model) and we study the evolution of the neutrino gas along the $z$-axis which can also be interpreted as being the radial direction in spherical coordinate. The mono-energetic $\nu_{e}$ and $\bar{\nu}_{e}$ beams $(\omega= \pm 1)$ are assumed to be emitted with $\mathbf{v}_{ \pm}=\left( \pm u, 0, v_{z}\right)$ where $u=\sqrt{1-v_{z}^{2}}$ with $v_{z}=1 / 2$ (corresponding to an opening angle of $2 \pi / 3$ between the two beams) and the ratio between the number densities is fixed to be $n_{\bar{\nu}_{e}} / n_{\nu_{e}}=0.7$.

We solve Eq. (7) for our stationary model $(\Omega=0)$ to find unstable modes in the $z$-direction (where the imaginary part of $k_{z}$ is positive) and the $E-\mathbf{v}$ distributions of the corresponding eigenvectors as a function of the Fourier mode in the $x$-direction, $k$ (hereafter we drop the subscripts $x$ and superscripts $\Omega$ ). In addition, for the small turbulence amplitudes considered here one can safely ignore the turbulence in the $z$ (longitudinal) direction since it is completely suppressed by the other terms in the equation of motion. This implies that Fourier modes are only coupled in the $x$ (transverse) direction. Note that a similar suppression does not exist for the turbulence in the $x$-direction since there is no other term being able to compete with the turbulence (coupling) effect.

To illustrate how turbulent matter density fluctuations impact the stability of a dense neutrino medium, in Fig. 1 we indicate the overall shape of the eigenvectors of Eq. (7), defined as

$$
\left|Q^{k}\right|=\left(\sum_{E, \mathbf{v}}\left|Q_{E, \mathbf{v}}^{k}\right|^{2}\right)^{1 / 2}
$$

corresponding to the unstable mode with the maximum growth rate, where the eigenvectors are normalized to have unit length. In the left panel, we first consider a neutrino gas with a relatively low neutrino number density, $\mu=\sqrt{2} G_{\mathrm{F}} n_{\nu_{e}}=50 \mathrm{~km}^{-1}$. For such values of $\mu$, only very low Fourier modes are unstable in a homogenous neutrino gas. However, the instability structure changes dramatically in a turbulent medium. As expected, there is a dominant
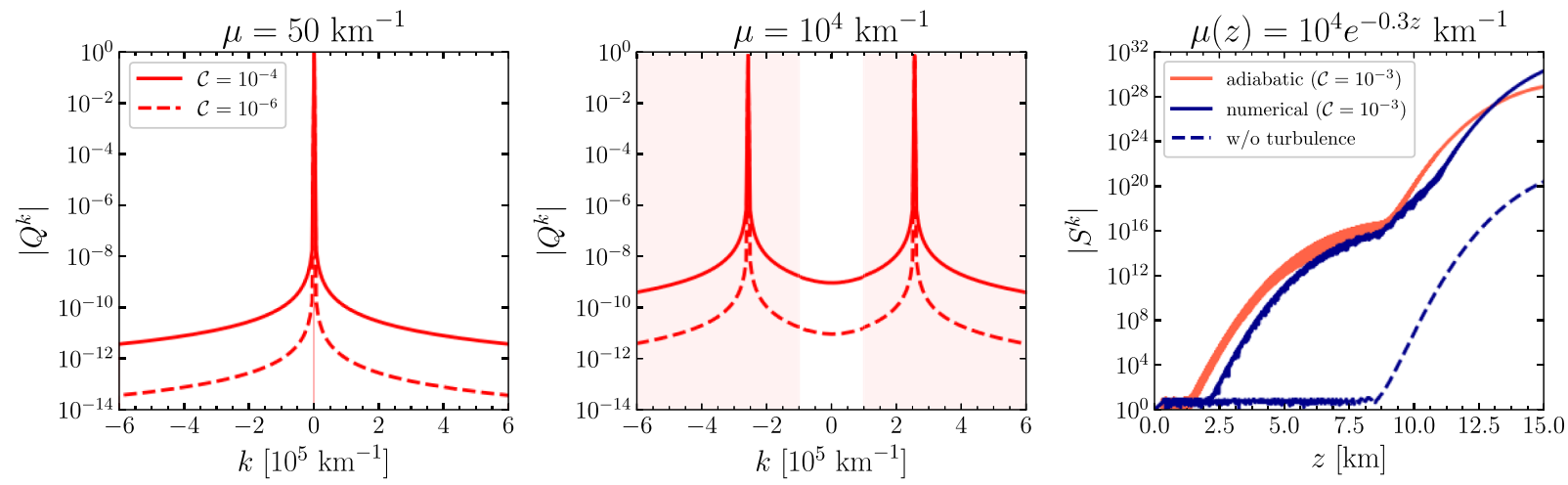

FIG. 1. Left and middle panels: overall shape of the eigenvectors of Eq. (7), corresponding to the unstable mode with the maximum growth rate for two representative values of $\mu$ and for $\mathcal{C}=10^{-4}$ and $\mathcal{C}=10^{-6}$. The shaded area indicates the unstable region in the homogenous case (which is extremely narrow in the left panel). Here, we have used Eq. (12) to relate matter to neutrino number density. Right panel: evolution of the flavor coherence term in the linear regime [Eq. (4)] for the representative $k=10^{3} \mathrm{~km}^{-1}$ Fourier mode in a declining $\mu$. Here, we solve Eq. (4) numerically for a discrete set of Fourier modes with $\Delta k=10^{3} \mathrm{~km}^{-1}$ and assuming $\lambda_{0}=300 \mu$. 
peak with $\left|Q^{k}\right| \simeq 1$ at $k \simeq 0$. But due to the presence of turbulent matter fluctuations, one can clearly see that the instability can now leak to much higher Fourier modes (and make them unstable) which are otherwise completely stable in the homogenous case. It is of utmost importance to note that in spite of its small amplitude (for the tiny turbulence amplitudes used here), the leakage of instabilities can entirely change the stability condition of the neutrino gas. This simply comes from the fact that as far as the flavor stability is concerned, the amplitude (of the unstable modes) is not relevant since any unstable mode can growth exponentially with a growth rate of $\sim 10 \mathrm{~km}^{-1}$ (for slow modes). Thus, even unstable modes with amplitude $\left|Q^{k}\right| \sim 10^{-9}$ can get activated within only $\sim 2 \mathrm{~km}$. This implies that, surprisingly, even a tiny amount of turbulence in matter would be enough to have a notable influence and make the whole range of (relevant) Fourier modes unstable with reasonable initial amplitudes. This is very different from the turbulence-induced parametric resonances where turbulent matter fluctuations can only generate a noticeable effect when the turbulence amplitude is considerably large [65]. To the best of our knowledge, the flavor instability leakage is the only physical effect in CCSNe which is sensitive to such tiny turbulence amplitudes. Indeed, the turbulence effect behaves here like a switch-on effect. Thus, one might be tempted to interpret the leakage phenomenon as an example of the effect of the background symmetry breaking in a dense neutrino gas. Note that in the absence of turbulence, $\left|Q^{k}\right|$ should be a $\delta$ function in the Fourier space.

The turbulence-induced leakage amplitude is almost independent of $\mu$ and depends only on the density fluctuation amplitude (see Appendix A)

$$
\text { leakage of } k_{0} \rightarrow k_{0} \pm k: \frac{\left|Q^{k_{0} \pm k}\right|}{\left|Q^{k_{0}}\right|} \sim \frac{\lambda_{k}}{k} \text {. }
$$

By using this expression, one can easily make an estimate of the leakage amplitude for a given matter density and turbulence amplitude.

In the middle panel of Fig. 1, an example of the instability leakage for a high neutrino number density with $\mu=10^{4} \mathrm{~km}^{-1}$ is presented. For such a value of $\mu$ which is expected in the SN zones close to the surface of the PNS, only very large $k$ 's are unstable in the homogenous case. However, the instability leaks to small $k$ 's in the presence of turbulence. In particular, the leakage amplitude for a given turbulence amplitude is much larger in this case since the matter density is quite big in the vicinity of the PNS.

Although the form of the eigenvectors of Eq. (7) changes significantly in a turbulent medium, its eigenvalues do not change noticeably, at least for such small turbulence amplitudes tried here. This implies that this novel effect observed for constant $\mu$ 's might be still superficial unless it can also leave its influence on the solutions of Eq. (4) for a realistic SN profile where $\mu$ is changing. But this is exactly where the power of the leakage mechanism is best manifested, as illustrated in the right panel of Fig. 1. Here to provide a flavor of this effect, we show the evolution of the $k=10^{3} \mathrm{~km}^{-1}$ Fourier mode in a model in which the neutrino number density is varying with $\mu(z)=10^{4} \exp (-0.3 z) \mathrm{km}^{-1}$ (note that $\mu$ changes very rapidly and goes from $10^{4}$ to $10 \mathrm{~km}^{-1}$ within only $\sim 20 \mathrm{~km}$ ). As can be clearly seen, the final amplitude of the Fourier modes (at the point they become dominant) in the presence of turbulence can be larger than those of the homogenous gas by many orders of magnitude. This is due to the fact that all the relevant Fourier modes can always grow exponentially in a turbulent medium in contrast to the homogenous gas in which each Fourier mode has a certain range of instability. This behavior is completely compatible with/predictable from what already observed in the left and middle panels of Fig. 1 and shows that the assessment based on the shape of the eigenvectors of Eq. (7) can be very useful in providing a sufficient insight on how Fourier modes grow. Note that the exact turbulence-induced enhancement in the amplitude of a Fourier mode depends on the duration on which the turbulence influences its evolution, which can be much longer for realistic SN profiles [89].

The evolution of the neutrino gas here is adiabatic to a very good degree in the sense that the scales on which the eigenvectors of Eq. (7) grow (exponentially) are much shorter than those of variations in $\mu$ (or in the shape of the eigenvectors themselves), i.e., $\kappa^{-1} \ll \mu /(\mathrm{d} \mu / \mathrm{d} r)$. One can then better understand the behavior observed in the right panel of Fig. 1 in an analytical way, assuming a perfect adiabaticity. In the perfect adiabatic limit, the solution of Eq. (4) at each step $z=z_{0}+\Delta z$ can be obtained analytically from the one at $z=z_{0}$ by $S\left(z_{0}+\Delta z\right)=$ $\sum_{i} c_{i} \Psi_{i} e^{i\left(k_{z}\right)_{i} \Delta z}$ where $\Psi_{i}$ and $\left(k_{z}\right)_{i}$ are the eigenvectors (which form a complete basis) and eigenvalues of the Hamiltonian of Eq. (7) at $z=z_{0}$ and $c_{i}$ 's are the expansion coefficients of $S\left(z_{0}\right)=\sum_{i} c_{i} \Psi_{i}$. Such an analytical adiabatic solution (red curve) shows a very good agreement with the numerical solution of Eq. (4). One should note that the key point here is that the eigenvectors of Eq. (7) at two different steps are not exactly linearly independent of each other. In other words, each $\Psi_{i}^{\text {new }}$ has contributions from all $\Psi_{j}^{\text {old }}$,s, i.e., $\Psi_{i}^{\text {new }}=\sum_{j} c_{i j} \Psi_{j}^{\text {old }}$ with $c_{i j}$ being roughly the turbulence amplitude. This means that any unstable mode grows from an enhanced initial value (occurred during the growth of the modes which were previously unstable) which in turn ensures that all the Fourier modes always grow exponentially during the propagation of neutrinos. This is entirely in contrast to the homogenous case where the new unstable modes at each point are totally linearly independent of the old ones at a previous point and therefore, any exponential growth is present only within a certain period. 


\section{DISCUSSION AND CONCLUSIONS}

The turbulence-induced leakage of flavor instabilities implies that the notion of $\mu-k$ instability band (see, e.g., Fig. 3 in Ref. [22]) developed in a homogenous neutrino gas is not very useful in a turbulent medium where what distinguishes different Fourier modes is actually only their initial amplitudes, $\left|Q^{k}\right|$.

One can immediately observe that the leakage mechanism can well address one of the biggest issues with slow instabilities in the deepest SN zones. In particular, it dismisses the necessity of the occurrence of fast modes in order to observe significant flavor conversions near the PNS. To demonstrate this idea, in Fig. 2 we show the instability footprints of two representative Fourier modes as a function of the distance from the SN core and the turbulence amplitude, $\mathcal{C}$, during the accretion phase of a CCSN. ${ }^{1}$ Here we take a matter/neutrino density profile approximately similar to that of Ref. [25] in which

$$
\mu(r)=\mu_{R}(R / r)^{4} \quad \text { and } \quad \lambda(r)=\lambda_{R}(R / r)^{3},
$$

where $\mu_{R}$ and $\lambda_{R}$ are the neutrino and matter densities on the surface of the neutrinosphere, $R$, respectively, for which we have used $R=15 \mathrm{~km}, \mu_{R}=2 \times 10^{5} \mathrm{~km}^{-1}$ and $\lambda_{R}=6 \times 10^{7} \mathrm{~km}^{-1}$ (corresponding to a matter density of $\rho \simeq 3 \times 10^{11} \mathrm{~g} \mathrm{~cm}^{-3}$ ). For very small turbulence amplitudes, the instability zones can be extremely narrow specially at small radii which prevents any significant flavor conversions therein. However, as $\mathcal{C}$ increases, the Fourier modes become unstable at all radii which means that they can grow many orders of magnitude (as in the right panel of Fig. 1) and easily enter the nonlinear regime. Hence, no matter whether fast modes exist or not, collective neutrino oscillations can occur within just a few km above the PNS. In addition, unlike fast modes which can only exist in small SN regions and are less likely to occur in nonexploding models, turbulence-induced flavor conversion modes are ubiquitous and generic. This could have an important impact on the SN dynamics and the nucleosynthesis of heavy elements in CCSNe.

Apart from the crucial impact of turbulence on the flavor stability of a dense neutrino gas, its presence is also important in providing initial seeds for the unstable modes. Specifically, the turbulence term in Eq. (4) transfers the initial seed from $k_{0}$ to $k_{0} \pm k$ on scales $\sim \lambda_{k}^{-1}$, or more accurately, $\sim \max \left\{\lambda_{k^{\prime}}^{-1} k / k^{\prime}\right\}$ where the maximum is taken over all turbulence modes.

\footnotetext{
${ }^{1}$ Here we define the unstable region for a given Fourier mode by requiring $\left|Q^{k}\right|>10^{-13}$ for the eigenvector of the mode with maximum growth rate. This is to ensure that the activation scale of a given mode is shorter than the variation scales of $\mu$. Otherwise, such boundaries for the unstable regions are absolutely artificial.
}

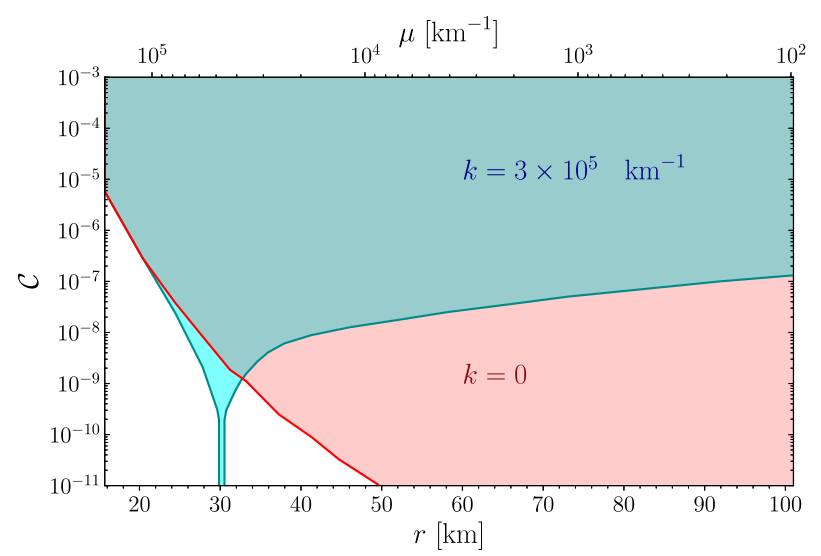

FIG. 2. Instability regions (shaded areas) of two representative Fourier modes, $k=0$ and $3 \times 10^{5} \mathrm{~km}^{-1}$, as a function of the distance from the SN core and the turbulence amplitude. Here we have employed the two-beam model described in the text.

Apart from its impact on slow modes which was discussed here, turbulence can also affect fast neutrino flavor conversion modes (see Appendix C). However, although a similar leakage can occur therein, the leakage mechanism does not seem to change DR of fast modes.

In the above discussions, we have only considered the effects of spatial density fluctuations on the spatial instabilities. However, a similar effect should also be expected for temporal instabilities as shown in Appendix B. Indeed, the leakage effect has nothing to do with the eigenvalues of DR equation and the nature of instabilities and, it only arises due to the presence of coupling among different eigenvectors. Additionally as discussed in Appendix C, such an effect even exists for stable solutions (real eigenvalues of DR equation). Similarly, temporal fluctuations of the matter density can also couple different temporal frequencies. Although extremely rapid temporal density variations are necessary to observe any noticeable effect, it could be still plausible considering the small required turbulence amplitudes. Moreover, while we have only considered the effects of the turbulence on flavor instability in CCSNe, similar effect can be expected in any dense neutrino environment where matter density fluctuations are present, such as neutron star merger remnant accretion disks.

Our study is meant only to serve as an introduction to this novel issue and further research is necessary to provide a better understanding of its physical implications. This is yet another time that the rich physics of neutrino flavor evolution in dense neutrino media surprises us.

\section{ACKNOWLEDGMENTS}

I am deeply indebted to Georg Raffelt and Huaiyu Duan for their comments on the manuscript, their kind encouragements and many insightful discussions during the development of this work. I am also grateful to Cristina 
Volpe, Ernazar Abdikamalov and Shashank Shalgar for useful conversations/communications and to Hans-Thomas Janka and Francesco Capozzi for reading the manuscript and their comments. I acknowledge partial support by the Deutsche Forschungsgemeinschaft (DFG) through Grant No. SFB 1258 (Collaborative Research Center Neutrinos, Dark Matter, Messengers).

\section{APPENDIX A: THE LEAKAGE AMPLITUDE}

We here develop a hand-waving understanding of the turbulence-induced leakage amplitude. We take the structure of the equation of neutrino flavor evolution in the linear regime and attempt to understand how the presence of a coupling between different Fourier modes changes the structure of the eigenvectors. For this purpose, we consider the following set of equations which resembles the evolution of the flavor coherence terms of two Fourier modes which are coupled

$$
\begin{aligned}
& i \partial_{t} S^{k_{1}}=\left(\omega+\mu+k_{1}\right) S^{k_{1}}+\lambda_{k} S^{k_{2}} \\
& i \partial_{t} S^{k_{2}}=\left(\omega+\mu+k_{2}\right) S^{k_{2}}+\lambda_{k} S^{k_{1}}
\end{aligned}
$$

where $k=k_{2}-k_{1}$. It can be easily shown that the eigenvectors of this set of linear equations have the form $|Q| \propto\left(1, \lambda_{k} / k\right)$ and $\left(\lambda_{k} / k, 1\right)$ for $\lambda_{k} / k \ll 1$ (which is always the case for the Fourier modes of interest). This confirms that in the presence of a coupling term, any eigenvector will have a dominant component at a given Fourier mode and subdominant contributions from other modes with amplitude $\sim \lambda_{k} / k$.

\section{APPENDIX B: TEMPORAL INSTABILITIES}

We have studied the leakage mechanism in a stationary dense neutrino gas. Here, we demonstrate that a very similar effect arises in a time-dependent model where turbulence can impact the temporal instabilities. This should be of course expected since the leakage effect has nothing to do with the eigenvalues of DR equation and the nature of instabilities and, it only arises due to the presence of coupling among different eigenvectors. Hence, as will also be discussed in our upcoming work [89], the leakage effect exists also for multiangle configurations.

We here consider a time-dependent two-beam, monochromatic neutrino gas with one spatial dimension. Our model is the same as the one proposed in Ref. [40] but we only consider two (zenith) angle beams with emission angles $\vartheta_{1}=\pi / 6$ and $\vartheta_{2}=\pi / 3$ with respect to the $z$-axis. We here assume that the neutrino gas possesses a perfect axial symmetry about the $z$-axis.

The results obtained in this model are presented in Fig. 3 where we have as an example shown the overall shape of $\left|Q^{k}\right|$ (here $\mathrm{k}$ is the Fourier mode in $z$-direction) for $\mu=50$, corresponding to the unstable temporal mode with the maximum growth rate. As can be obviously seen, the

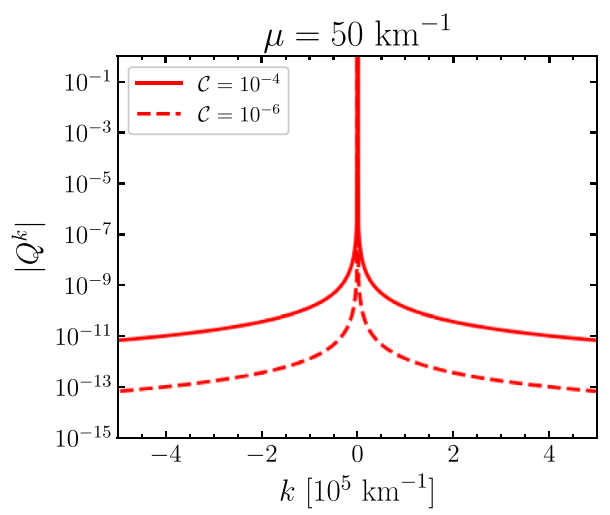

FIG. 3. Overall shape of the eigenvectors (of Eq. (7) in the main text) for the time-dependent neutrino gas described here, corresponding to the unstable temporal mode with the maximum growth rate for $\mu=50 \mathrm{~km}^{-1}$. Here to relate matter to neutrino number density we have used Eq. (12) in the main text.

temporal instabilities are similarly affected by the leakage mechanism.

\section{APPENDIX C: FAST MODES}

Turbulent matter fluctuations can also influence fast modes in a similar way to slow modes. This is clearly illustrated in Fig. 4 where two arbitrary stable and unstable modes are shown for a neutrino gas in the presence of fast modes. Here we have considered a 2D stationary neutrino gas with only one neutrino and one antineutrino beams with $\mathbf{v}_{\bar{\nu}_{e}}=\left(+u, 0, v_{z}\right)$ and $\mathbf{v}_{\nu_{e}}=\left(-u, 0, v_{z}\right)\left(v_{z}=1 / 2\right)$ in such a way that fast modes can exist (see the model studied in Ref. [33]). The blue and red bands indicate the regions where the real branches and the gap (where complex branches exist) are located, respectively, in a homogenous neutrino gas. In the presence of turbulence, both the real

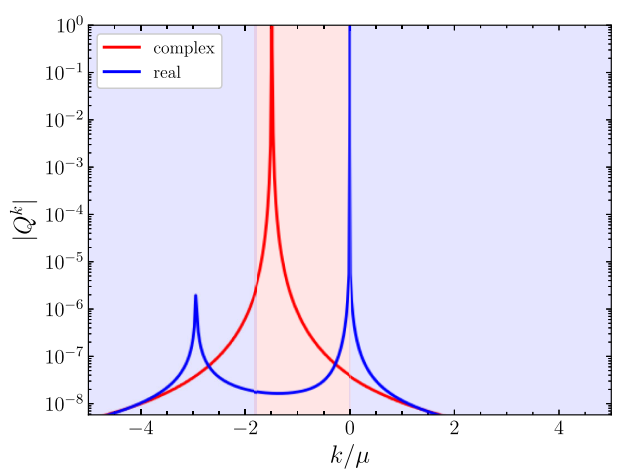

FIG. 4. Overall shape of two arbitrary real and complex solutions (of Eq. (7) in the main text), in the presence of fast modes in a turbulent medium, with $\mathcal{C}=10^{-6}$ and assuming $\lambda=300 \mu$. The blue and red areas show the regions where the real branches and the gap (the complex branches) exist in a homogenous neutrino gas, respectively. 
and complex solutions can leak to the other zone. Note that the leakage phenomenon is not unique to the unstable modes (complex branches) and stable modes also leak to the unstable region (with $\left|Q^{k}\right| \sim \lambda_{k} / k$ ). Note, however, that considering the locality of fast modes, as long as the eigenvalues dispersion relation (DR) equation are not modified by the turbulence one can always define a new set of basis so that the DR remains unchanged.
[1] H.-T. Janka, Explosion mechanisms of core-collapse supernovae, Annu. Rev. Nucl. Part. Sci. 62, 407 (2012).

[2] A. Burrows, Colloquium: Perspectives on core-collapse supernova theory, Rev. Mod. Phys. 85, 245 (2013).

[3] S. Pastor and G. Raffelt, Flavor Oscillations in the Supernova Hot Bubble Region: Nonlinear Effects of Neutrino Background, Phys. Rev. Lett. 89, 191101 (2002).

[4] H. Duan, G. M. Fuller, J. Carlson, and Y.-Z. Qian, Simulation of coherent non-linear neutrino flavor transformation in the supernova environment. 1. Correlated neutrino trajectories, Phys. Rev. D 74, 105014 (2006).

[5] H. Duan, G. M. Fuller, J. Carlson, and Y.-Z. Qian, Coherent Development of Neutrino Flavor in the Supernova Environment, Phys. Rev. Lett. 97, 241101 (2006).

[6] H. Duan, G. M. Fuller, and Y.-Z. Qian, Collective neutrino oscillations, Annu. Rev. Nucl. Part. Sci. 60, 569 (2010).

[7] Y.Z. Qian and S. E. Woosley, Nucleosynthesis in neutrino driven winds: 1. The physical conditions, Astrophys. J. 471, 331 (1996).

[8] K. Scholberg, Supernova neutrino detection, Annu. Rev. Nucl. Part. Sci. 62, 81 (2012).

[9] A. Gallo Rosso, S. Abbar, F. Vissani, and M. C. Volpe, Late time supernova neutrino signal and proto-neutron star radius, J. Cosmol. Astropart. Phys. 12 (2018) 006.

[10] J. F. Beacom, The diffuse supernova neutrino background, Annu. Rev. Nucl. Part. Sci. 60, 439 (2010).

[11] H. Duan, G. M. Fuller, J. Carlson, and Y.-Z. Qian, Flavor Evolution of the Neutronization Neutrino Burst from an O-Ne-Mg Core-Collapse Supernova, Phys. Rev. Lett. 100, 021101 (2008).

[12] B. Dasgupta, A. Dighe, G. G. Raffelt, and A. Y. Smirnov, Multiple Spectral Splits of Supernova Neutrinos, Phys. Rev. Lett. 103, 051105 (2009).

[13] S. Galais and C. Volpe, The neutrino spectral split in corecollapse supernovae: A magnetic resonance phenomenon, Phys. Rev. D 84, 085005 (2011).

[14] H. Duan, G. M. Fuller, J. Carlson, and Y.-Z. Qian, Neutrino Mass Hierarchy and Stepwise Spectral Swapping of Supernova Neutrino Flavors, Phys. Rev. Lett. 99, 241802 (2007).

[15] H. Duan, Collective neutrino oscillations and spontaneous symmetry breaking, Int. J. Mod. Phys. E 24, 1541008 (2015).

[16] H. Duan and A. Friedland, Self-Induced Suppression of Collective Neutrino Oscillations in a Supernova, Phys. Rev. Lett. 106, 091101 (2011).

[17] S. Sarikas, G. G. Raffelt, L. Hudepohl, and H.-T. Janka, Suppression of Self-Induced Flavor Conversion in the Supernova Accretion Phase, Phys. Rev. Lett. 108, 061101 (2012).
[18] S. Chakraborty, T. Fischer, A. Mirizzi, N. Saviano, and R. Tomas, No Collective Neutrino Flavor Conversions During the Supernova Accretion Phase, Phys. Rev. Lett. 107, 151101 (2011).

[19] G. Raffelt, S. Sarikas, and D. d. S. Seixas, Axial Symmetry Breaking in Self-Induced Flavor Conversion of Supernova Neutrino Fluxes, Phys. Rev. Lett. 111, 091101 (2013).

[20] H. Duan, Flavor oscillation modes in dense neutrino media, Phys. Rev. D 88, 125008 (2013).

[21] H. Duan and S. Shalgar, Flavor instabilities in the neutrino line model, Phys. Lett. B 747, 139 (2015).

[22] S. Abbar, H. Duan, and S. Shalgar, Flavor instabilities in the multiangle neutrino line model, Phys. Rev. D 92, 065019 (2015).

[23] S. Abbar and H. Duan, Neutrino flavor instabilities in a time-dependent supernova model, Phys. Lett. B 751, 43 (2015).

[24] S. Chakraborty, R. S. Hansen, I. Izaguirre, and G. Raffelt, Self-induced flavor conversion of supernova neutrinos on small scales, J. Cosmol. Astropart. Phys. 01 (2016) 028.

[25] S. Chakraborty, R. Hansen, I. Izaguirre, and G. Raffelt, Collective neutrino flavor conversion: Recent developments, Nucl. Phys. B908, 366 (2016).

[26] A. Mirizzi, G. Mangano, and N. Saviano, Self-induced flavor instabilities of a dense neutrino stream in a twodimensional model, Phys. Rev. D 92, 021702 (2015).

[27] J. D. Martin, S. Abbar, and H. Duan, Nonlinear flavor development of a two-dimensional neutrino gas, Phys. Rev. D 100, 023016 (2019).

[28] G. Mangano, A. Mirizzi, and N. Saviano, Damping the neutrino flavor pendulum by breaking homogeneity, Phys. Rev. D 89, 073017 (2014).

[29] J. D. Martin, J. Carlson, and H. Duan, Spectral swaps in a two-dimensional neutrino ring model, Phys. Rev. D 101, 023007 (2020).

[30] R. F. Sawyer, Speed-up of neutrino transformations in a supernova environment, Phys. Rev. D 72, 045003 (2005).

[31] R. F. Sawyer, Neutrino Cloud Instabilities Just Above the Neutrino Sphere of a Supernova, Phys. Rev. Lett. 116, 081101 (2016).

[32] S. Chakraborty, R. S. Hansen, I. Izaguirre, and G. Raffelt, Self-induced neutrino flavor conversion without flavor mixing, J. Cosmol. Astropart. Phys. 03 (2016) 042.

[33] I. Izaguirre, G. Raffelt, and I. Tamborra, Fast Pairwise Conversion of Supernova Neutrinos: A Dispersion-Relation Approach, Phys. Rev. Lett. 118, 021101 (2017).

[34] M.-R. Wu and I. Tamborra, Fast neutrino conversions: Ubiquitous in compact binary merger remnants, Phys. Rev. D 95, 103007 (2017). 
[35] F. Capozzi, B. Dasgupta, E. Lisi, A. Marrone, and A. Mirizzi, Fast flavor conversions of supernova neutrinos: Classifying instabilities via dispersion relations, Phys. Rev. D 96, 043016 (2017).

[36] S. A. Richers, G. C. McLaughlin, J. P. Kneller, and A. Vlasenko, Neutrino quantum kinetics in compact objects, Phys. Rev. D 99, 123014 (2019).

[37] S. Abbar and H. Duan, Fast neutrino flavor conversion: Roles of dense matter and spectrum crossing, Phys. Rev. D 98, 043014 (2018).

[38] S. Abbar and M. C. Volpe, On fast neutrino flavor conversion modes in the nonlinear regime, Phys. Lett. B 790, 545 (2019).

[39] F. Capozzi, B. Dasgupta, A. Mirizzi, M. Sen, and G. Sigl, Collisional Triggering of Fast Flavor Conversions of Supernova Neutrinos, Phys. Rev. Lett. 122, 091101 (2019).

[40] J. D. Martin, C. Yi, and H. Duan, Dynamic fast flavor oscillation waves in dense neutrino gases, Phys. Lett. B 800, 135088 (2020).

[41] F. Capozzi, G. Raffelt, and T. Stirner, Fast neutrino flavor conversion: Collective motion vs decoherence, J. Cosmol. Astropart. Phys. 09 (2019) 002.

[42] C. Dring, R. S. L. Hansen, and M. Lindner, Stability of three neutrino flavor conversion in supernovae, J. Cosmol. Astropart. Phys. 08 (2019) 003.

[43] L. Johns, H. Nagakura, G. M. Fuller, and A. Burrows, Neutrino oscillations in supernovae: Angular moments and fast instabilities, Phys. Rev. D 101, 043009 (2020).

[44] S. Shalgar, I. Padilla-Gay, and I. Tamborra, Neutrino propagation hinders fast pairwise flavor conversions, J. Cosmol. Astropart. Phys. 06 (2020) 048.

[45] J. F. Cherry, G. M. Fuller, S. Horiuchi, K. Kotake, T. Takiwaki, and T. Fischer, Time of flight and supernova progenitor effects on the neutrino halo, Phys. Rev. D 102, 023022 (2020).

[46] M. Chakraborty and S. Chakraborty, Three flavor neutrino conversions in supernovae: Slow and fast instabilities, J. Cosmol. Astropart. Phys. 01 (2020) 005.

[47] S. Abbar, Searching for fast neutrino flavor conversion modes in core-collapse supernova simulations, J. Cosmol. Astropart. Phys. 05 (2020) 027.

[48] F. Capozzi, M. Chakraborty, S. Chakraborty, and M. Sen, Fast Flavor Conversions in Supernovae: The Rise of Mu-Tau Neutrinos, Phys. Rev. Lett. 125, 251801 (2020).

[49] Z. Xiong, A. Sieverding, M. Sen, and Y.-Z. Qian, Potential impact of fast flavor oscillations on neutrino-driven winds and their nucleosynthesis, arXiv:2006.11414.

[50] S. Bhattacharyya and B. Dasgupta, Fast neutrino flavor conversion at late time, Phys. Rev. D 102, 063018 (2020).

[51] S. Shalgar and I. Tamborra, Dispelling a myth on dense neutrino media: Fast pairwise conversions depend on energy, J. Cosmol. Astropart. Phys. 01 (2021) 014.

[52] S. Abbar, H. Duan, K. Sumiyoshi, T. Takiwaki, and M. C. Volpe, On the occurrence of fast neutrino flavor conversions in multidimensional supernova models, Phys. Rev. D 100, 043004 (2019).

[53] S. Abbar, H. Duan, K. Sumiyoshi, T. Takiwaki, and M. C. Volpe, Fast neutrino flavor conversion modes in multidimensional core-collapse supernova models: The role of the asymmetric neutrino distributions, Phys. Rev. D 101, 043016 (2020).

[54] M. Delfan Azari, S. Yamada, T. Morinaga, H. Nagakura, S. Furusawa, A. Harada, H. Okawa, W. Iwakami, and K. Sumiyoshi, Fast collective neutrino oscillations inside the neutrino sphere in core-collapse supernovae, Phys. Rev. D 101, 023018 (2020).

[55] H. Nagakura, T. Morinaga, C. Kato, and S. Yamada, Fastpairwise collective neutrino oscillations associated with asymmetric neutrino emissions in core-collapse supernova, arXiv:1910.04288.

[56] T. Morinaga, H. Nagakura, C. Kato, and S. Yamada, Fast neutrino-flavor conversion in the preshock region of corecollapse supernovae, Phys. Rev. Research 2, 012046 (2020).

[57] R. Glas, H. T. Janka, F. Capozzi, M. Sen, B. Dasgupta, A. Mirizzi, and G. Sigl, Fast neutrino flavor instability in the neutron-star convection layer of three-dimensional supernova models, Phys. Rev. D 101, 063001 (2020).

[58] S. Abbar, F. Capozzi, R. Glas, H. T. Janka, and I. Tamborra, On the characteristics of fast neutrino flavor instabilities in three-dimensional core-collapse supernova models, arXiv:2012.06594.

[59] E. Abdikamalov, C. D. Ott, D. Radice, L. F. Roberts, R. Haas, C. Reisswig, P. Msta, H. Klion, and E. Schnetter, Neutrino-driven turbulent convection and standing accretion shock instability in three-dimensional core-collapse supernovae, Astrophys. J. 808, 70 (2015).

[60] Q. Mabanta and J. W. Murphy, How turbulence enables core-collapse supernova explosions, Astrophys. J. 856, 22 (2018).

[61] S. M. Couch and C.D. Ott, The role of turbulence in neutrino-driven core-collapse supernova explosions, Astrophys. J. 799, 5 (2015).

[62] D. Radice, E. Abdikamalov, C. D. Ott, P. Msta, S. M. Couch, and L.F. Roberts, Turbulence in core-collapse supernovae, J. Phys. G 45, 053003 (2018).

[63] C. A. Meakin and D. Arnett, Turbulent convection in stellar interiors. 1. Hydrodynamic simulation, Astrophys. J. 667, 448 (2007).

[64] L. Ma, S. Shalgar, and H. Duan, Matter parametric neutrino flavor transformation through Rabi resonances, Phys. Rev. D 98, 103011 (2018).

[65] T. Lund and J. P. Kneller, Combining collective, MSW, and turbulence effects in supernova neutrino flavor evolution, Phys. Rev. D 88, 023008 (2013).

[66] G. Reid, J. Adams, and S. Seunarine, Collective neutrino oscillations in turbulent backgrounds, Phys. Rev. D 84, 085023 (2011).

[67] G. L. Fogli, E. Lisi, A. Mirizzi, and D. Montanino, Damping of supernova neutrino transitions in stochastic shock-wave density profiles, J. Cosmol. Astropart. Phys. 06 (2006) 012.

[68] J. F. Cherry, M.-R. Wu, J. Carlson, H. Duan, G. M. Fuller, and Y.-Z. Qian, Density fluctuation effects on collective neutrino oscillations in $\mathrm{O}-\mathrm{Ne}-\mathrm{Mg}$ core-collapse supernovae, Phys. Rev. D 84, 105034 (2011).

[69] K. M. Patton, J. P. Kneller, and G. C. McLaughlin, Stimulated neutrino transformation through turbulence on a changing density profile and application to supernovae, Phys. Rev. D 91, 025001 (2015). 
[70] Y. Yang and J.P. Kneller, Neutrino flavour evolution through fluctuating matter, J. Phys. G 45, 045201 (2018).

[71] A. Friedland and A. Gruzinov, Neutrino signatures of supernova turbulence, arXiv:astro-ph/0607244.

[72] J. P. Kneller, G. C. McLaughlin, and J. Brockman, Oscillation effects and time variation of the supernova neutrino signal, Phys. Rev. D 77, 045023 (2008).

[73] J. P. Kneller and C. Volpe, Turbulence effects on supernova neutrinos, Phys. Rev. D 82, 123004 (2010).

[74] E. Borriello, S. Chakraborty, H.-T. Janka, E. Lisi, and A. Mirizzi, Turbulence patterns and neutrino flavor transitions in high-resolution supernova models, J. Cosmol. Astropart. Phys. 11 (2014) 030.

[75] G. Sigl and G. Raffelt, General kinetic description of relativistic mixed neutrinos, Nucl. Phys. B406, 423 (1993).

[76] P. Strack and A. Burrows, Generalized Boltzmann formalism for oscillating neutrinos, Phys. Rev. D 71, 093004 (2005).

[77] C. Y. Cardall, Liouville equations for neutrino distribution matrices, Phys. Rev. D 78, 085017 (2008).

[78] C. Volpe, D. Vnnen, and C. Espinoza, Extended evolution equations for neutrino propagation in astrophysical and cosmological environments, Phys. Rev. D 87, 113010 (2013).

[79] A. Vlasenko, G. M. Fuller, and V. Cirigliano, Neutrino quantum kinetics, Phys. Rev. D 89, 105004 (2014).
[80] L. Wolfenstein, Neutrino oscillations in matter, Phys. Rev. D 17, 2369 (1978).

[81] S. P. Mikheyev and A. Yu. Smirnov, Resonance amplification of oscillations in matter and spectroscopy of solar neutrinos, Sov. J. Nucl. Phys. 42, 913 (1985); 42, 305 (1986).

[82] G. M. Fuller, R. W. Mayle, J. R. Wilson, and D. N. Schramm, Resonant neutrino oscillations and stellar collapse, Astrophys. J. 322, 795 (1987).

[83] D. Nötzold and G. Raffelt, Neutrono dispersion at finite temperature and density, Nucl. Phys. B307, 924 (1988).

[84] J. T. Pantaleone, Dirac neutrinos in dense matter, Phys. Rev. D 46, 510 (1992).

[85] A. Banerjee, A. Dighe, and G. Raffelt, Linearized flavorstability analysis of dense neutrino streams, Phys. Rev. D 84, 053013 (2011).

[86] D. Vnnen and C. Volpe, Linearizing neutrino evolution equations including neutrino-antineutrino pairing correlations, Phys. Rev. D 88, 065003 (2013).

[87] S. Airen, F. Capozzi, S. Chakraborty, B. Dasgupta, G. Raffelt, and T. Stirner, Normal-mode analysis for collective neutrino oscillations, J. Cosmol. Astropart. Phys. 12 (2018) 019.

[88] U. Frisch and A. Kolmogorov, Turbulence: The legacy of AN Kolmogorov.

[89] S. Abbar (to be published). 